\title{
Clima de segurança do paciente em um hospital especializado em oncologia*
}

\section{Patient safety climate in a hospital specialized in oncology}

\author{
Maria Helena Barbosa ${ }^{1}$, Eduarda Mendes Sousa ${ }^{2}$, Márcia Marques dos Santos Felix ${ }^{3}$,
} Karoline Faria Oliveira ${ }^{4}$, Elizabeth Barichello ${ }^{5}$

\footnotetext{
* Trabalho obteve apoio financeiro da Fundação de Amparo à Pesquisa do Estado de Minas Gerais.

${ }^{1}$ Enfermeira, Doutora em Enfermagem na Saúde do Adulto. Professor Associado da Universidade Federal do Triângulo Mineiro (UFTM). Uberaba, MG, Brasil. E-mail: mhelena331@hotmail.com.

${ }^{2}$ Discente do curso de graduação em Enfermagem da UFTM. Uberaba, MG, Brasil. E-mail: eduardams08@hotmail.com.

${ }^{3}$ Enfermeira, Mestre em Enfermagem Fundamental. Discente do Programa de Pós-Graduação em Atenção à Saúde, nível Doutorado, da UFTM. Uberaba, MG, Brasil. E-mail: mm-sf@hotmail.com.

${ }^{4}$ Enfermeira, Mestre em Atenção à Saúde. Discente do Programa de Pós-Graduação em Atenção à Saúde, nível Doutorado, da UFTM. Uberaba, MG, Brasil. Email: karol_fmtm2005@yahoo.com.br.

${ }^{5}$ Enfermeira, Doutora em Enfermagem. Professor Adjunto da UFTM. Uberaba, MG, Brasil. E-mail: lizabarichello@yahoo.com.br.
}

\section{RESUMO}

O objetivo do estudo foi avaliar o clima de segurança na perspectiva dos profissionais da equipe de saúde de um hospital especializado em oncologia. Estudo observacional, seccional, realizado com 66 profissionais da saúde, utilizando-se o Safety Attitudes Questionnaire. Para as análises foram utilizados teste $t$ de Student e correlação de Sperman ( $\alpha=0,05)$. O escore geral do instrumento foi 70,28. O domínio com melhor escore foi satisfação no trabalho $(86,74)$ e os domínios com menor escore foram percepção da gerência $(64,99)$ e percepção do estresse $(61,74)$. Não houve diferenças estatisticamente significativa das médias entre os sexos, mas esta esteve presente entre os que realizaram ou não pósgraduação. Não houve correlação entre escores e tempo na especialidade de atuação na instituição. A avaliação final demonstrou fragilidades na percepção dos profissionais da saúde em relação às questões que envolvem o clima de segurança institucional.

Descritores: Segurança do Paciente; Oncologia; Serviço Hospitalar de Oncologia; Gestão da Segurança.

\section{ABSTRACT}

The study's objective was to assess the safety climate from the perspective of a health team from a hospital specialized in oncology. An observational sectional study, conducted with 66 health professionals, using the Safety Attitudes Questionnaire. For analysis, Student's t test and Sperman's correlation ( $\alpha=0.05$ ) were used. The instrument's general score was 70.28. The domain with best score was satisfaction at work (86.74) and, the domains with lower scores were perception from management (64.99) and stress perception (61.74). There was no differences of means statistically significant between genders, but it was present between those who had gone through graduate school or not. There was no correlation between scores and career time in the specialty at the institution. The final assessment demonstrated fragilities in the perception of health professionals related to questions involving the institutional climate of safety.

Descriptors: Patient Safety; Medical Oncology; Oncology Service, Hospital; Safety Management. 


\section{INTRODUÇÃO}

O termo "cultura de segurança" pode ser definido como o conjunto de valores, atitudes e percepções, tanto individuais como de grupos, que determinam o compromisso e o estilo quanto às questões voltadas para a segurança do paciente em uma organização de saúde ${ }^{(1)}$.

Proporcionar segurança é de extrema relevância para aqueles que prestam o serviço e para aqueles que fazem uso do mesmo. Para haver um melhor clima de segurança, é necessário que um indivíduo ou um grupo de pessoas estabeleça valores, competências e atitudes, que gerem esse ambiente seguro, reduzindo ao mínimo aceitável o risco de danos desnecessários durante a atenção à saúde $\mathrm{e}^{(2-3)}$.

Dimensões do clima de segurança têm sido associadas a diversos desfechos de saúde, como a ocorrência de eventos adversos, ou seja, incidentes indesejáveis e que podem ser prevenidos, que ocorrem durante a prestação do cuidado à saúde. Esses eventos resultam em danos relacionados aos pacientes, como incapacidades, traumas físicos e psicológicos, aumento no tempo de permanência hospitalar, afastamento do convívio social e do trabalho, além de prejuízos éticos e morais, para os profissionais de saúde ${ }^{(4)}$. As instituições de saúde também são acometidas com o aumento dos custos, perda da confiança na instituição, e danos morais e organizacionais ${ }^{(5)}$.

A segurança do paciente tornou-se uma grande prioridade para os articuladores políticos, profissionais de saúde e gestores. Monitorar a segurança de um hospital é uma tarefa um tanto quanto desafiadora. Para tal, é necessário conhecer o perfil institucional, com o intuito de observar aspectos que desfavoreçam a implementação de um ambiente com opiniões que compreendam ações seguras e atuar sobre esses aspectos, para proporcionar uma assistência planejada e de qualidade ${ }^{(3,6)}$.

No hospital especializado, aquele destinado a prestar cuidados a pacientes que necessitam de assistência voltada para uma determinada especialidade médica, como o serviço hospitalar de oncologia, a tarefa pode ser ainda mais difícil. Isso porque o câncer é uma doença complexa, que pode ser de longa duração e que compromete significativamente a vida dos indivíduos nas dimensões biológica, social e afetiva, exigindo assistência especializada de diferentes profissionais ${ }^{(7)}$. 0 planejamento de ações seguras a esses pacientes reduziria a possibilidade de ocorrência de eventos adversos relacionados às vulnerabilidades que pacientes oncológicos perpassam durante seu tratamento.

Verifica-se a escassez de estudos nacionais, com a finalidade de mensurar o clima de segurança de instituições hospitalares. Entre os instrumentos utilizados para avaliar atitudes de segurança, o Safety Attitudes Questionnaire (SAQ), validado no Brasil em 2012, ainda tem sido pouco adotado, embora se trate de um instrumento confiável e considerado um dos mais sensíveis para essa avaliação ${ }^{(8)}$. Observa-se, em geral, a utilização de outros instrumentos de avaliação de segurança, como em estudo realizado no Estado do Paraná, com a aplicação da escala denominada Clima de Segurança, traduzida e validada no Brasil ${ }^{(9)}$.

Diante do exposto, o presente estudo teve como objetivo avaliar o clima de segurança do paciente, na perspectiva dos profissionais da equipe de saúde, em um hospital especializado em oncologia.

\section{MÉTODOS}

Estudo observacional, seccional, com abordagem quantitativa, realizado em um hospital especializado em oncologia, situado em um município localizado na região do Triângulo Mineiro (MG), Brasil.

A população alvo (N) deste estudo foi constituída por 107 profissionais da equipe de saúde contratados, dentre os quais estavam a equipe de enfermagem (enfermeiros, técnicos em enfermagem e auxiliares em enfermagem), médicos, nutricionistas, farmacêuticos, psicólogos, fisioterapeutas, assistentes sociais, técnicos em farmácia, laboratório e radiologia. Como critérios de inclusão, foram considerados: estarem lotados em suas unidades 
de atuação há mais de um mês e possuir regime de trabalho mínimo de 20 horas semanais.

Dos 107 profissionais (N), 23 foram excluídos do estudo por se encontrarem afastados do trabalho durante o período de coleta de dados e 18 se recusaram participar da pesquisa. Assim, a amostra ( $n$ ) constituiu-se de 66 profissionais. A coleta de dados ocorreu durante o período de junho a setembro de 2013.

Para obtenção dos dados, utilizou-se o SAQ, validado para o idioma português do Brasil ${ }^{(10)}$, o qual mensura o clima de segurança percebido pelos profissionais da saúde, que reflete diretamente o clima de segurança da organização. O SAQ foi entregue aos profissionais da equipe de saúde para serem preenchidos e devolvidos posteriormente, com data de devolução preestabelecida, conforme recomendação para a aplicação deste instrumento.

Foram obtidas também as variáveis sociodemográficas e profissionais, a saber: sexo, categoria profissional, tempo de atuação na especialidade e a unidade de atuação, atuação principal e profissional, tempo de formação e de atuação na instituição, pós-graduação, presença de outro vínculo empregatício e vínculo institucional. Esses dados constavam de um instrumento estruturado, o qual foi entregue juntamente do SAQ para devolução na mesma data pré-agendada para devolução.

Todos os participantes assinaram o Termo de Consentimento Livre e Esclarecido.

O instrumento SAQ contém 41 itens, sendo 38 correspondentes à seis domínios: (1) Clima de Trabalho em Equipe, considerado como a qualidade do relacionamento e a colaboração entre os membros de uma equipe (itens 1 a 6); (2) Clima de Segurança, que considera a percepção dos profissionais quanto ao comprometimento organizacional para segurança do paciente (itens 7 a 13); (3) Satisfação no Trabalho, sobre a visão positiva do local de trabalho (itens 15 a 19); (4) Percepção do Estresse, reconhecimento de quanto os fatores estressores que podem influenciar na execução do trabalho (itens 20 a 23); (5) Percepção da Gerência, sobre a aprovação das ações da gerência ou administração, tanto da unidade em que o profissional atua, quanto do hospital como um todo (itens 24 a 29); e (6) Condições de Trabalho, que considera a percepção da qualidade do ambiente de trabalho (itens 30 a 33) ${ }^{(11)}$.

Os itens 14, 34 a 36 não fazem parte de nenhum domínio no instrumento original. A resposta de cada item segue a escala de cinco pontos do tipo Likert: "discorda totalmente" (A), "discorda parcialmente" (B), "neutro" (C), "concorda parcialmente" (D), "concorda totalmente" (E) e "não se aplica". O escore final da escala varia de zero a 100. Valores maior ou igual a 75 pontos são considerados avaliação positiva ${ }^{(10)}$. Para critérios de interpretação dos dados deste estudo, foi utilizado como nivelamento o valor de pontuação sugerido pelos idealizadores do instrumento, considerando que tais dados não foram testados no Brasil.

Os dados foram inseridos em uma planilha eletrônica do programa Excel para Windows ${ }^{\circledR}$, validados por dupla digitação e exportados para o programa Statistical Package for the Social Sciences (SPSS), versão 19.0 para Windows ${ }^{\circledR}$, para processamento e análise.

As variáveis qualitativas foram analisadas segundo estatística descritiva por meio da distribuição de frequência absoluta e porcentual, enquanto para as variáveis quantitativas foram utilizadas medidas descritivas de centralidade (média) e de dispersão (desvio padrão, valor mínimo e valor máximo).

Para análise bivariada, da influência das variáveis categóricas sobre os escores de segurança, utilizou-se o teste $t$ de Student (categóricas dicotômicas) e o teste de correlação de Spearman, para as variáveis ordinais. Foram consideradas associações estatisticamente significativas aquelas com $\mathrm{p} \leq 0,05$.

Este estudo faz parte do projeto de pesquisa intitulado $A$ cultura de segurança do paciente em hospitais de uma região de Minas Gerais, que teve apoio financeiro da Fundação de Amparo à Pesquisa do Estado de Minas Gerais (FAPEMIG). Foi aprovado pelo Comitê de 
Ética em Pesquisa com Seres Humanos da Universidade Federal do Triângulo Mineiro (CEP-UFTM), parecer $n$ 은 2.306, respeitando a legislação brasileira para pesquisas envolvendo seres humanos.

\section{RESULTADOS}

Participaram do estudo 66 profissionais de saúde, sendo 19 (28,8\%) técnicos em enfermagem, 15 (22,7\%) técnicos em farmácia, laboratório e radiologia, 12 (18,2\%) fisioterapeutas, cinco $(7,6 \%)$ enfermeiros, quatro $(6,1 \%)$ farmacêuticos, quatro $(6,1 \%)$ psicólogos, dois $(3,0 \%)$ assistentes sociais, um (1,5\%) auxiliar de enfermagem e um $(1,5 \%)$ nutricionista. Destes, $46(69,7 \%)$ eram do sexo feminino, $26(39,4 \%)$ possuíam de cinco a 10 anos de formação, sendo que a maioria $(62,1 \%)$ não possuía pósgraduação. Dos 25 (37,9\%) que possuíam, 23 (34,8\%) era do tipo especialização, um $(1,5 \%)$ mestrado e um $(1,5 \%)$ doutorado.

Em relação ao tempo em que atuavam na instituição, $15(22,7 \%)$ profissionais atuavam por um período de um a dois anos no hospital especializado em oncologia. 0 mesmo período foi encontrado para 16 (24,2\%) profissionais em relação à sua atuação na especialidade encontrada. A maioria dos profissionais $(68,2 \%)$ possuía atuação em cargos somente assistenciais e 51,5\% atuavam apenas com adultos.

Quanto ao setor de atuação, o maior número de profissionais trabalhava em área semicrítica, com prevalência do setor de Clínica Médica, com 23 (34,8\%) profissionais. A área crítica foi a segunda com maior número de profissionais, sendo sete $(10,6 \%)$ profissionais lotados na unidade de terapia intensiva (UTI) adulto. Na área não crítica, a maioria trabalhava na Comissão de Controle de Infecção Hospitalar (CCIH) e no Departamento de Serviço Social, com 3\% cada. Do total de participantes do estudo, 39 (59,1\%) possuíam apenas um vínculo empregatício.

A Tabela 1 apresenta as características sociodemográficas e profissionais dos participantes do estudo.
A Tabela 2 apresenta os escores de cada um dos seis domínios do clima de segurança, conforme o SAQ. O escore geral médio obtido pelo instrumento foi de 70,28 $(S=14,25)$, com mínimo de 27,44 e máximo de 97,97 . 0 domínio que apresentou maior escore foi o três, relacionado a satisfação no trabalho, tendo média de $86,74(\mathrm{~S}=14,79)$, afirmando que a maioria dos profissionais estava satisfeita com o local de trabalho. 0 domínio que apresentou o menor escore esteve relacionado à percepção do estresse, domínio quatro, com média de $61,74(S=27,45)$.

A Tabela 3 apresenta as respostas dos itens 14, 33, 34, 35 e 36 - aqueles que não pertencem a nenhum domínio. Desse modo, a maioria das respostas esteve concentrada em "concordo parcialmente" e "concordo totalmente".

A questão 33 ("Eu vivencio boa colaboração com os(as) enfermeiros(as) nesta área") foi a que mais apresentou índice de resposta para "concordo totalmente": 33 (50,0\%). A partir das respostas, observou-se que havia um entrosamento positivo entre os profissionais, as equipes e a administração.

Em relação à análise bivariada, não houve diferença entre os sexos $(p>0,05)$; mas houve diferença em relação a ter ou não realizado pós-graduação no escore geral $(p=0,01)$, domínio um $(p=0,03)$, domínio três $(p=0,04)$, domínio cinco $(p=0,01)$ e domínio seis $(p=0,02)$.

Não houve correlação com o tempo em que atuava na instituição, tempo na especialidade e tempo de formação com os escores ( $p>0,05)$, exceto em relação ao tempo na especialidade com o domínio quatro (coeficiente de correlação 0,29, p=0,01). 
Tabela 1: Características sociodemográficas e profissionais dos participantes da pesquisa. Uberaba, MG, Brasil, 2013.

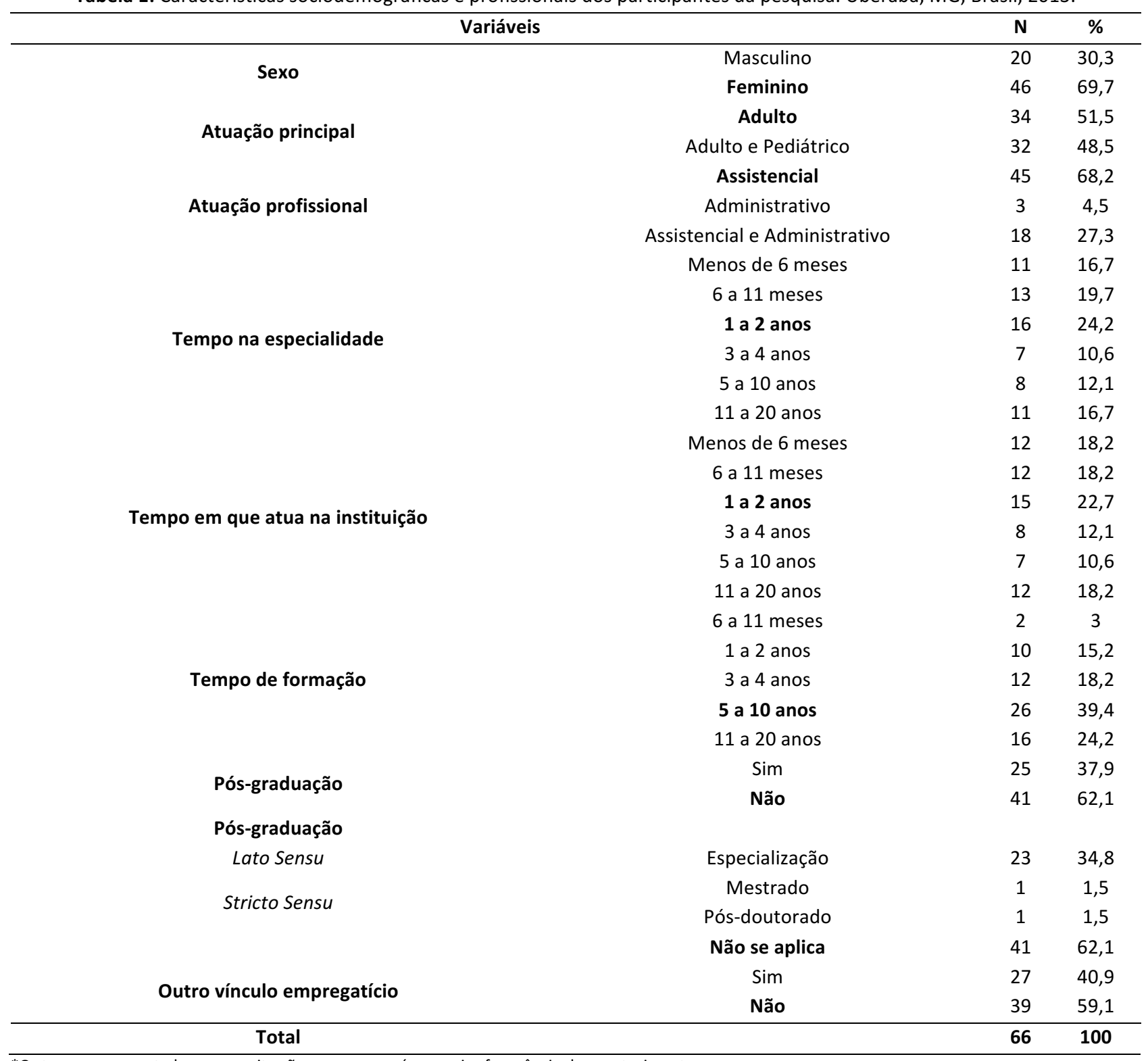

*Os termos apresentados em negrito são os que possuíram maior frequência de acontecimentos.

Tabela 2: Distribuição da análise dos escores por domínios. Uberaba, MG, Brasil, 2013.

\begin{tabular}{lccccc}
\hline Domínios & $\begin{array}{c}\text { Clima de Trabalho } \\
\text { em Equipe }\end{array}$ & $\begin{array}{c}\text { Clima de } \\
\text { Segurança }\end{array}$ & $\begin{array}{c}\text { Satisfação no } \\
\text { Trabalho }\end{array}$ & $\begin{array}{c}\text { Percepção do } \\
\text { Estresse }\end{array}$ & $\begin{array}{c}\text { Percepção da } \\
\text { Gerência }\end{array}$ \\
\hline $\begin{array}{l}\text { Média } \\
\text { Desvio }\end{array}$ & 73,91 & 72,00 & 86,74 & 61,74 & 64,99 \\
padrão & 19,31 & 17,75 & 14,79 & 27,45 & 18,88 \\
Mínima & 25,00 & 32,14 & 30,00 & 0,00 & 13,64 \\
Máxima & 100,00 & 100,00 & 100,00 & 100,00 & 100,00 \\
\hline
\end{tabular}


Tabela 3: Frequência de respostas dos participantes relacionada aos itens que não corresponderam a nenhum domínio. Uberaba, MG, Brasil, 2013.

\begin{tabular}{|c|c|c|c|c|c|c|}
\hline \multirow{2}{*}{ Itens não pertencentes a nenhum domínio } & DT & DP & Neutro & $\mathbf{C P}$ & CT & NA \\
\hline & $\mathrm{n}(\%)$ & $\mathrm{n}(\%)$ & $n(\%)$ & $n(\%)$ & $\mathrm{n}(\%)$ & $n(\%)$ \\
\hline 14. Minhas sugestões sobre segurança seriam postas em ação se eu as & 3 & 7 & 21 & 17 & 15 & 3 \\
\hline expressasse à administração. & $-4,5$ & $-10,6$ & $-31,9$ & $-25,7$ & $-22,7$ & $-4,6$ \\
\hline \multirow{2}{*}{ 33. Eu vivencio boa colaboração com os(as) enfermeiros(as) nesta área. } & \multirow{2}{*}{-} & 2 & 8 & 24 & 28 & 4 \\
\hline & & -3 & $-12,1$ & $-36,4$ & $-42,5$ & -6 \\
\hline \multirow{2}{*}{ 34. Eu vivencio boa colaboração com a equipe de médicos nesta área. } & \multirow[b]{2}{*}{ - } & 3 & 9 & 19 & 33 & 1 \\
\hline & & $-4,5$ & $-13,6$ & $-28,8$ & -50 & $-1,5$ \\
\hline \multirow{2}{*}{ 35. Eu vivencio boa colaboração com os farmacêuticos nesta área. } & 1 & 4 & 12 & 15 & 25 & 9 \\
\hline & $-1,5$ & $-6,1$ & $-18,2$ & $-22,7$ & $-37,9$ & $-13,6$ \\
\hline \multirow{2}{*}{$\begin{array}{l}\text { 36. (R) Falhas na comunicação que levam a atrasos no atendimento são } \\
\text { comuns. }\end{array}$} & 7 & 5 & 10 & 22 & 20 & 2 \\
\hline & $-10,6$ & $-7,6$ & $-15,2$ & $-33,3$ & $-30,3$ & -3 \\
\hline
\end{tabular}

DT: discordo totalmente; DP: discordo parcialmente; CP: concordo parcialmente; CT: concordo totalmente; NA: não se aplica; R: tem reverso.

\section{DISCUSSÃO}

Dos 66 profissionais que responderam ao instrumento, prevaleceu a equipe de enfermagem, com $25(37,9 \%)$ profissionais, com maior proporção entre os técnicos de enfermagem (28,8\%). A maior adesão dos profissionais ter sido marcada pela equipe de enfermagem pode ser vista como ponto positivo, pois são profissionais que estão envolvidos em período contínuo na prática assistencial e gerencial do paciente ${ }^{(12)}$. Além de o enfermeiro ser responsável por avaliar o paciente bem como os riscos que ameaçam sua segurança ${ }^{(13)}$.

A maioria dos profissionais $(69,7 \%)$ era do sexo feminino, como também foi encontrado em estudo desenvolvido em Taiwan $(87,2 \%)^{(14)}$. Um dos motivos das mulheres estarem em maior número é pela representatividade feminina na equipe de enfermagem. Outra justificativa é que a Enfermagem, Nutrição, Serviço Social, Psicologia e Letras aparecem como as carreiras que se apresentam destinadas às mulheres ${ }^{(15)}$, e quatro dessas profissões citadas participaram desta pesquisa. $\mathrm{Na}$ literatura, estudos realizados em unidades de terapia intensiva, utilizando o mesmo instrumento, também obtiveram maior proporção da equipe de enfermagem $(82 \% \text { e } 41,6 \% \text { respectivamente) })^{(16-17)}$.

Quanto ao tempo na especialidade dos profissionais e ao tempo de atuação/experiência em seu respectivo setor, houve maior proporção entre um e dois anos de atuação $(24,2 \%)$, período que coincide com o tempo de atuação na instituição para $22,7 \%$ dos profissionais. Referente ao tempo de formação profissional, nota-se maior número de profissionais com cinco a 10 anos de formação $(39,4 \%)$ e sem pós-graduação $(62,1 \%)$.

Um bom desempenho na execução dos procedimentos gerais da profissão pode ser reflexo do tempo de experiência do profissional, porém aqueles profissionais que apresentam menor tempo de experiência tendem a maiores chances de discutirem suas dificuldades com aqueles com mais anos de experiência, fornecendo percepções distintas do clima de segurança na instituição(18).

Observou-se que $62,1 \%$ dos profissionais não possuíam pós-graduação. 0 mercado de trabalho exige qualificações e especializações que tragam um diferencial e valorizem os profissionais. A busca de capacitações atualiza o conhecimento, que é dinâmico na área da saúde, como o uso de novas tecnologias ou evidências novas em relação a tratamentos e terapias.

A maioria dos profissionais $(59,1 \%)$, não possuía outro vínculo empregatício. A dupla jornada de trabalho favorece a diminuição do tempo dedicado ao autocuidado e ao lazer, potencializando o cansaço e estresse $^{(19)}$.

Em relação aos escores do domínio $S A Q$, o estudo apresentou um escore geral médio de 70,28 ( $S=14,25)$ pontos, ou seja, menor do que o recomendado na metodologia. A literatura corrobora os dados do presente 
estudo, com valor abaixo do recomendado $(61,5$ pontos) $)^{(10)}$.

Em relação ao domínio 1, considerando a qualidade do relacionamento e a colaboração entre os membros de uma equipe, o escore médio foi de $73,91 \quad(S=19,31)$ pontos, ou seja, valores inferiores ao recomendado. Outros estudos também encontraram médias inferiores a 75,0 para este domínio ${ }^{(14,20)}$. O bom relacionamento em equipe favorece a qualidade da assistência e contribui de forma significativa para atitudes de segurança.

Dentre os componentes que influenciam positivamente no clima de segurança, estão o aprendizado organizacional, a comunicação sobre os erros e o trabalho em equipe. Para assegurar uma baixa insatisfação no contexto de trabalho, um bom relacionamento no ambiente de trabalho precisa ser garantido $^{(4)}$. Em estudo realizado em hospitais da Jordânia observou-se uma relação forte e positiva entre o clima de segurança e o trabalho em equipe ${ }^{(21)}$.

O domínio 2, clima de segurança, obteve média 72,00 $(S=17,74)$ pontos, demonstrando também uma percepção negativa em relação ao comprometimento organizacional para segurança do paciente. Dentre alguns estudos que utilizaram o SAQ, foram encontradas médias superiores para este domínio, que variaram entre 80,4 e $70,9^{(16,20)}$. Poucos estudos foram encontrados com médias do clima de segurança inferiores ${ }^{(14,22)}$.

Cabe ressaltar que instituições com altos níveis de clima de segurança tendem a apresentar menores índices de ocorrência de eventos adversos, maior notificação de erros e incidentes, melhor comunicação entre os gestores e funcionários e maior segurança do paciente ${ }^{(23)}$.

O domínio 3, satisfação no trabalho, apresentou a maior média $(86,74 ; S=14,79)$, demonstrando que os profissionais deste estudo estavam satisfeitos nessa área. Corroborando esses resultados, estudo realizado em um hospital no interior de São Paulo registrou que $94 \%$ dos profissionais de saúde afirmaram que gostavam do trabalho que realizavam ${ }^{(11)}$. A qualidade da assistência prestada está relacionada diretamente com a satisfação do profissional em seu trabalho.

Quanto à percepção do estresse, no domínio 4, a maioria dos profissionais deste estudo não reconheceu o quanto os fatores estressores interferiam na execução do trabalho, pois o escore geral desse domínio foi de 61,74 $(S=27,45)$, representando o menor escore entre todos os domínios. Fatores como carga excessiva de trabalho, e situações tensas e de cansaço podem comprometer de maneira séria a segurança do paciente e gerar circunstâncias prejudiciais ao paciente. Corroborando tal resultado, foram encontradas médias inferiores a 75,00 na literatura ${ }^{(16,20)}$.

No domínio 5, percepção da gerência da unidade e do hospital, observou-se média de 64,98 ( $S=18,87)$, demonstrando uma visão negativa dos profissionais do estudo quanto às ações da gerência com relação às questões de segurança. Este domínio de percepção da gerência pelo profissional reflete a conformidade do profissional quanto às ações da gerência ou da administração do hospital e da unidade relacionadas à segurança do paciente ${ }^{(11)}$. Em estudo realizado na Suíça, a média para esse domínio foi de 70,25 ( $S=21,60)$, demonstrando também uma visão negativa dos profissionais de saúde investigados ${ }^{(24)}$.

Referente ao domínio 6, condições de trabalho, observou-se média geral de 67,23 ( $\mathrm{S}=26,86)$, representando uma visão negativa dos profissionais quanto à percepção da qualidade de suporte ambiental e logístico no local de trabalho, ao contrário de outros estudos, em que os autores observaram médias superiores às do presente estudo para este domínio ${ }^{(16,20)}$.

O excesso de cargas de trabalho é visto como responsável pelo desgaste emocional, ocorrência de acidentes e problemas de saúde dos profissionais. Deve existir um planejamento adequado da distribuição de carga de trabalho, educação continuada, elaboração de estratégias para melhoria das condições de trabalho, com o intuito de prevenir o desgaste físico e psíquico da equipe $^{(25)}$. 
Quanto aos itens que não correspondem a nenhum domínio, o item 14 pergunta se o profissional acredita que suas sugestões sobre segurança seriam postas em ação se ele as expressasse à administração. Apenas 22,7\% dos profissionais investigados concordaram totalmente com essa questão, demonstrando uma visão negativa em relação a esse item. Estudo brasileiro utilizando o SAQ encontrou que, de 203 profissionais, apenas 40\% concordaram totalmente quanto à participação de sua opinião na administração do hospital ${ }^{(11)}$.

Os outros itens dizem respeito à colaboração vivenciada entre profissionais e falhas na comunicação que levam a atrasos no atendimento. A comunicação, dentro das organizações hospitalares, pode influenciar na qualidade assistencial, bem como ações voltadas para segurança do paciente. Com maioria das respostas "concordo totalmente", pode-se notar que houve um entrosamento positivo entre os profissionais neste estudo, garantindo melhor assistência.

Estudo realizado com a equipe de enfermagem de um hospital de ensino obteve porcentagens superiores para os itens de colaboração entre a equipe, e $80 \%$ dos profissionais concordaram com a boa colaboração entre os enfermeiros, $73 \%$ com a dos médicos e $45 \%$ com a colaboração dos farmacêuticos da unidade ${ }^{(11)}$.

\section{REFERÊNCIAS}

1. Nieva VF, Sorra J. Safety culture assessment: a tool for improving patient safety in healthcare organizations. Qual Saf Heal Care [Internet]. 2003 [acesso em: $31 \mathrm{dez} .2015$ ];12(Suppl 2):ii17-23. Disponível em:

http://dx.doi.org/10.1136/qhc.12.suppl_2.ii17.

2. Wegner W, Pedro ENR. Patient safety in care circumstances: prevention of adverse events in the hospitalization of children. Rev Lat Am Enfermagem [Internet]. 2012 Jun [acesso em: 31 dez. 2015];20(3):427-34. Disponível em:

http://dx.doi.org/10.1590/S0104-11692012000300002.

3. Ques ÁAM, Montoro CH, González MG. Strengths and threats regarding the patient's safety: nursing professionals' opinion. Rev Lat Am Enfermagem [Internet]. 2010 Jun [acesso em: 31 dez. 2015];18(3):339-45. Disponível em:

http://dx.doi.org/10.1590/s0104-11692010000300007.

4. Alahmadi HA. Assessment of patient safety culture in Saudi Arabian hospitals. Qual Saf Health Care [Internet]. 2010 [acesso

\section{CONCLUSÃO}

O clima de segurança neste hospital especializado em oncologia apresentou fragilidades em cinco dos seis domínios avaliados, com avaliação positiva apenas no domínio 5, satisfação no trabalho. Ressalta-se a necessidade de maior atenção por parte da gerência hospitalar para os domínios "percepção do estresse", "percepção da gerência" e "condições de trabalho", pois as percepções dos profissionais investigados quanto a estes domínios foram as mais negativas.

Com os resultados deste estudo, deve ser possível ajudar no planejamento de ações que incentivem melhorias relacionadas ao clima de segurança institucional e que auxiliem na capacitação dos profissionais investigados, necessários para identificar e resolver causas sistêmicas subjacentes relacionadas com a segurança do paciente e a qualidade da assistência no serviço.

Para ser realmente eficaz, a segurança do paciente precisa ser incorporada à educação dos profissionais de saúde em todo o âmbito de cuidados de saúde, melhorando, assim, a percepção dos mesmos sobre as atitudes da organização quanto ao clima de segurança. Essa mudança requer esforços e envolvimento de toda a instituição.

em: 31 dez. 2015];19(5):e17. Disponível em: http://dx.doi.org/10.1136/qshc.2009.033258.

5. Carneiro FS, Bezerra ALQ, Silva AEBC, Souza LP, Paranaguá TTB, Branquinho NCSS. Eventos adversos na clínica cirúrgica de um hospital universitário: instrumento de avaliação da qualidade. 2010 [acesso em: 31 dez. 2015];19(2):204-11. Disponível em:

http://www.facenf.uerj.br/v19n2/v19n2a06.pdf. 6. El-Jardali F, Sheikh F, Garcia NA, Jamal D, Abdo A. Patient safety culture in a large teaching hospital in Riyadh: baseline assessment, comparative analysis and opportunities for improvement. BMC Health Serv Res [Internet]. 2014 [acesso em: 31 dez. 2015];14(1):122. Disponível em: http://dx.doi.org/10.1186/1472-6963-14-122. 7. Silva MEDC, Silva LDC, Dantas ALB, Araújo DOR, Duarte IS, Sousa JFM. Assistência de enfermagem ao paciente oncológico no hospital. Rev Enferm da UFPI [Internet]. 2014 [acesso em: 
31 dez. 2015];2(5):69-75. Disponível em:

http://www.ojs.ufpi.br/index.php/reufpi/article/view/1359.

8. Colla JB, Bracken AC, Kinney LM, Weeks WB.. Measuring patient safety climate: a review of surveys. Qual Saf Heal Care [Internet]. 2005 [acesso em: 31 dez. 2015];14(5):364-6. Disponível em:

http://dx.doi.org/10.1136\%2Fqshc.2005.014217.

9. Ribeiro PHV, Brevidelli MM, Tipple AFV, Ribeiro RP, Gir E. Clima de segurança organizacional e a adesão às precauções padrão entre dentistas. Acta Paul Enferm [Internet]. 2013 [acesso em: 31 dez. 2015];26(2):192-7. Disponível em: http://dx.doi.org/10.1590/S0103-21002013000200014.

10. Carvalho REFL, Cassiani SHDB. Cross-cultural adaptation of the Safety Attitudes Questionnaire - Short Form 2006 for Brazil. Rev Lat Am Enfermagem [Internet]. 2012 [acesso em: $31 \mathrm{dez}$. 2015];20(3):575-82. Disponível em:

http://dx.doi.org/10.1590/S0104-11692012000300020. 11. Rigobello MCG, Carvalho REFL, Cassiani SHB, Galon T, Capucho HC, Deus NN. The climate of patient safety: perception of nursing professionals. Acta Paul Enferm [Internet]. 2012 [acesso em: 31 dez. 2015];25(5):728-35. Disponível em: http://dx.doi.org/10.1590/S010321002012000500013.

12. Pereira MD, Souza DF, Ferraz F. Segurança do paciente nas ações de enfermagem hospitalar: uma revisão integrativa da literatura. Revista Inova Saúde [Internet]. 2014 [acesso em: 31 dez. 2015];3(2):55-87. Disponível em:

http://periodicos.unesc.net/Inovasaude/article/view/1746. 13. Silva DT, Goulart NS, Amado KC. Registros de enfermagem com ênfase na segurança do paciente. Rev Rede Cuid em Saúde [Internet]. 2014 [acesso em: 31 dez. 2015];8(2):1-4. Disponível em:

http://publicacoes.unigranrio.br/index.php/rcs/article/view/23 76.

14. Lee WC, Wung HY, Liao HH, Lo CM, Chang FL, Wang PC et al. Hospital safety culture in Taiwan: a nationwide survey using Chinese version Safety Attitude Questionnaire. BMC Health Serv Res [Internet]. 2010 [acesso em: 31 dez. 2015];10:234. Disponível em: http://dx.doi.org/10.1186\%2F1472-6963-10234.

15. Ramos MO, Ulbanere RC, Jesus BS. Mulheres no Mercado de Trabalho. Rev Científica Integr [Internet]. 2014 [acesso em: 31 dez. 2015];1(4). Disponível em:

http://www.unaerp.br/revista-cientifica-integrada/edicoesanteriores/edicao-n-4-2014-1-1/1498-432-1506-1-sm/file. 16. Profit J, Etchegaray J, Petersen LA, Sexton JB, Hysong SJ, Mei $M$ et al. The Safety Attitudes Questionnaire as a tool for benchmarking safety culture in the NICU. Arch Dis Child Fetal Neonatal Ed [Internet]. 2012 [acesso em: $31 \mathrm{dez}$.

2015];97(2):F127-32. Disponível em:

http://dx.doi.org/10.1136\%2Farchdischild-2011-300612.

17. Huang DT, Clermont G, Kong L, Weissfeld LA, Sexton JB et al. Intensive care unit safety culture and outcomes: a US multicenter study. Int J Qual Health Care [Internet]. 2010 [acesso em: $31 \mathrm{dez} .2015]$;22(3):151-61. Disponível em: http://dx.doi.org/10.1093\%2Fintqhc\%2Fmzq017.
18. Tomazoni A, Rocha PK, Souza S, Anders JC, Malfussi HFC. Patient safety culture at neonatal intensive care units: perspectives of the nursing and medical team. Rev Lat Am Enfermagem [Internet]. 2014 [acesso em: $31 \mathrm{dez}$. 2015];22(5):755-63. Disponível em: http://dx.doi.org/10.1590/0104-1169.3624.2477. 19. Araújo MDC, Barros CMP, Silva JS, Silva DG, Silva Filho MC. Pós-graduação: sua importância para o profissional de Secretariado Executivo. Revista do Secretariado Executivo, Passo Fundo [Internet]. 2013 [acesso em: 31 dez. 2015];9:13649. Disponível em:

http://www.upf.br/seer/index.php/ser/article/view/4039. 20. Poley MJ, van der Starre C, van den Bos A, van Dijk M, Tibboel $D$. Patient safety culture in a Dutch pediatric surgical intensive care unit: an evaluation using the Safety Attitudes Questionnaire. Pediatr Crit Care Med [Internet]. 2011 [acesso em: 31 dez. 2015];12(6):e310-6. Disponível em: http://dx.doi.org/10.1097/PCC.0b013e318220afca. 21. Abualrub RF, Gharaibeh HF, Bashayreh AE. The relationships between safety climate, teamwork, and intent to stay at work among Jordanian hospital nurses. Nurs Forum [Internet]. 2012 [acesso em: 31 dez. 2015];47(1):65-75. Disponível em: http://dx.doi.org/10.1111/j.17446198.2011.00253.x.

22. Gutiérrez-Cía I, Cos PM, Juan AY, Obón-Azuara B, AlonsoOvies Á, Martin-Delgado MC et al. Percepción de la cultura de seguridad en los servicios de medicina intensiva españoles. Med Clin (Barc) [Internet]. 2010 [acesso em: $31 \mathrm{dez}$. 2015];135:37-44. Disponível em: http://dx.doi.org/10.1016/S0025-7753(10)70019-1. 23. Hartmann CW, Meterko M, Rosen AK, Shibei Zhao, Shokeen $P$, Singer $S$ et al. Relationship of hospital organizational culture to patient safety climate in the Veterans Health Administration. Med Care Res Rev [Internet]. 2009 [acesso em: $31 \mathrm{dez}$. 2015];66(3):320-38. Disponível em: http://dx.doi.org/10.1177/1077558709331812.

24. Nordén-Hägg A, Sexton JB, Kälvemark-Sporrong S, Ring L, Kettis-Lindblad A. Assessing safety culture in pharmacies: the psychometric validation of the Safety Attitudes Questionnaire (SAQ) in a national sample of community pharmacies in Sweden. BMC Clin Pharmacol [Internet]. 2010 [acesso em: 31 dez. 2015];10:8. Disponível em: http://dx.doi.org/10.1186\%2F1472-6904-10-8. 25. Schmoeller R, Trindade LL, Neis MB, Gelbcke FL, Pires DEP. Cargas de trabalho e condições de trabalho da enfermagem: revisão integrativa. Rev Gaúcha Enferm [Internet]. 2011 [acesso em: 31 dez. 2015];32(2):368-77. Disponível em: http://dx.doi.org/10.1590/S1983-14472011000200022.

Recebido: 20/03/2015.

Aceito: 24/09/2015.

Publicado: 31/12/2015. 